\title{
The Safety of Peanut Oral Immunotherapy in Peanut Allergic Subjects in a Single-Center Trial
}

\author{
G.P. Yu, B. Weldon, S. Neale-May, and K.C. Nadeau \\ Division of Immunology and Allergy, Stanford University, Stanford, Calif., USA
}

\begin{abstract}
Background-Peanut allergy is the leading cause of food-related anaphylaxis and accidental exposures are common. Oral immunotherapy has been posited as a potential treatment.
\end{abstract}

Methods-Patients ages 3-65 with peanut-specific IgE $\geq 7 \mathrm{kU} / \mathrm{L}$ and/or a positive skin prick test with a history of an allergic reaction to peanut were recruited to undergo an oral immunotherapy protocol. All adverse reactions were recorded by research staff or patients in real time.

Results-Twenty-four patients received 6662 doses. Symptoms have been mostly mild (84\%) and only 3 severe gastrointestinal reactions required the administration of epinephrine. Abdominal pain has been the most common reaction, followed by oropharyngeal and lip pruritis. Respiratory symptoms have been rare.

Conclusions-In this trial of oral immunotherapy in adults and children, most reactions have been mild.

\section{Keywords}

food allergy; oral immunotherapy; peanut allergy

Peanut allergy, which affects an estimated $0.6 \%$ of U.S. adults and more than $1 \%$ of children, (1) is the leading cause of food related fatal anaphylaxis in the United States. (2) The standard of care is strict avoidance and readily available injectable epinephrine in case of accidental exposure, which is an unfortunately common occurrence with an estimated incidence of 0.33 exposures per patient year. (2) There is significant resource utilization in the form of ambulatory care and emergency room visits due to food related adverse events, estimated by Clark, et al. to be 203,000 ED visits per year from 2001 to 2005, and by Branum et al. to have averaged 317,000 ambulatory care and ED visits per year between 2004 and 2006 for food allergy-related diagnoses.

Oral immunotherapy (OIT) has been posited as a possible method of desensitization, with preliminary trials showing efficacy. (5-7) However, given that OIT relies on allergen ingestion on a daily basis at home, there have been concerns about safety. Jones, et al. reported reactions following $3.7 \%$ of home doses, 2 requiring epinephrine administration. Blumchen, et al. reported that $2.6 \%$ of buildup and home doses resulted in an objective adverse reaction. Most recently, in a randomized, placebo-controlled trial published by Varshney, et al., $47 \%$ of patients had clinically relevant adverse events during initial rush dosing, with two requiring epinephrine. Importantly, during build-up and home doses none

Correspondence to: Dr. Kari C. Nadeau, Division of Immunology and Allergy, Stanford University, 269 Campus Drive, CCSR Building, Room 2115, Stanford, CA 94305-5164 (USA), Tel. +1 650723 5227, knadeau@ stanford.edu.

Conflict of Interest

None 
of the peanut patients required epinephrine. To add to the safety database and to demonstrate for the first time safety in adults, we present preliminary safety data from an ongoing Phase 1 single-center trial of peanut OIT in adults and children.

\section{Methods}

Inclusion criteria included patients ages 3-65 years of age with a peanut-specific $\operatorname{IgE} \geq 7$ $\mathrm{kU} / \mathrm{L}$ and/or a positive peanut skin prick test and a history of an allergic reaction to peanut. (Table 1) Many of the subjects had had positive oral food challenges to peanut before entering the study. Patients underwent a modified rush day identical to a previously published protocol (5) where increasing amounts of peanut protein were ingested until the maximum dose (cumulative $12 \mathrm{mg}$ peanut protein) was reached or a reaction occurred. The following day, they were brought back to repeat the highest tolerated dose. If there was no reaction within an hour, they were sent home with daily doses. Patients were instructed to keep a daily diary of doses and any potential reactions with severity determined by subjective assessment (mild, moderate, or severe). Patients returned to the Clinical Translational Research Unit (CTRU) every two weeks where daily dosing diaries were reviewed and dose escalations occurred to a maximum maintenance dosage of 4,000 $\mathrm{mg}$ of peanut protein. Any reactions in the CTRU were graded based on Bock's criteria. (8) Research staff kept in close contact with patients and families to investigate any significant adverse events, and subjects had 24-hour contact information for all study personnel in case of a significant reaction or dosing question.

\section{Results}

Twenty-four subjects have received 6662 doses. Twenty-two of twenty-four patients have reported symptoms during either home doses or doses in the CTRU. Overall, there were 1023 symptoms recorded, $84 \%$ of which were mild and either self-resolved or resolved with antihistamines, $13 \%$ were moderate, and $3 \%$ were severe. Of the severe reactions, three, in separate patients, were treated with epinephrine. One was given in the CTRU following a dose escalation due to severe abdominal pain not responsive to multiple doses of antihistamines. Two were given at home following previously tolerated doses: 1) one with severe abdominal pain following an episode of diarrhea, and 2) the second was following multiple episodes of emesis with moderate throat pain unresponsive to antihistamines. For all three subjects, a single injection of epinephrine improved the clinical symptoms within a few minutes. None of these reactions resulted in discontinuation of therapy. All home reactions requiring epinephrine were associated with either exercise or bathing following the dose of peanut.

Any patients who experienced a moderate to severe reaction had their next dose decreased to a dose that was previously tolerated without any adverse symptoms. These patients were also asked to premedicate with $\mathrm{H} 1+/-\mathrm{H} 2$ antihistamines. These two strategies worked well to prevent and attenuate adverse reactions. However, one patient (subject F) while on $12 \mathrm{mg}$ of peanut protein had persistent abdominal and cough. The parents were not interested in premedicating or treating with antihistamines and the patient dropped out of the study. Of all of the symptoms in all subjects, abdominal pain has been the most common symptom (34\%), followed by oropharyngeal pruritus (25\%) and lip pruritus (9\%). (Figure 1) Eleven percent of total symptoms occurred in the CTRU at Stanford and $89 \%$ occurred at home. On average $13.6 \%$ of home doses per patient (range $0-74 \%$ ) resulted in allergic reactions.

Although not statistically significant due to a small sample size, there seemed to be an increased percentage of dose administrations resulting in reactions around the $25 \mathrm{mg}$ to $50 \mathrm{mg}$ dose levels. (Figure 2) These reactions were generally mild, resolving spontaneously or with 
antihistamines, though some delayed dose advancement at the next clinic visit. Although not statistically significant because of the small sample size, there were no differences in reaction severity or frequency between the adult $(n=2)$ and pediatric $(n=22)$ patients.

\section{Discussion}

The safety of OIT for peanut allergic patients has long been a concern in the design and implementation of trial protocols. (10-14) Since OIT necessitates ingesting the offending food, and ingestion of peanut allergen is responsible for more anaphylactic deaths than any other food allergen, it is easy to understand the trepidation of doctors and patients. However, peanut allergy is also a disease without an available disease-modifying treatment, and we live in a society where accidental exposures are almost unavoidable.

Several well-conducted pilot studies have indicated that although respiratory reactions to carefully administered peanut OIT occur, they may not be frequent, $(5-7,9)$ and our data supports the hypothesis that peanut OIT can be done safely. These data must be viewed with scrutiny, however, given the small sample sizes. Key components of safely administering an OIT regimen include experienced study personnel, extensive patient education, adherent patients and families, and ready access to life-saving therapies at the study site and at home.

As has recently been published, OIT is not yet ready to be considered the standard of care, and should only be conducted within the rigors of an IRB approved and monitored research study. $(10,14)$ Several aspects of OIT need to be understood prior to routine implementation. More research is needed to further delineate how to individualize treatment, which patients must be treated with the most caution, which can move through the dosing ladder with greater speed, and how to best prevent reactions. Certain dosing levels may be more likely to cause reactions, or may function as "threshold" doses, a dose level at which an individual subject has more reactions. Escalations may need to be slowed around these dose levels, and may be able to be advanced more quickly once higher levels are reached. The optimal goal dose for OIT should be investigated. Furthermore, more biochemical data is needed to help practitioners understand which patients will only become desensitized, thereby necessitating ongoing daily dosing, and which will become tolerant to peanuts, and how to know when an individual has crossed from the former into the latter category.

There are several ongoing studies looking at the safety and efficacy of peanut OIT in mostly pediatric populations. Our study is ongoing in both adults and children, and further phase 2 and 3 studies are needed to obtain optimal methods for best clinical practices and doses for peanut OIT.

\section{Acknowledgments}

We would like to acknowledge our sponsors - the Fund for Food Allergies, the NIH Clinical Translational Science Award (CTSA) and the Children's Health Research Program (CHRP) grant at Stanford, as well as the Lucile Packard Children's Hospital Foundation - without whose support this research would not have been possible. Our thanks to Dr. Wesley Burks, Dr. Stacie Jones, Dr. Mark Genovese, Dr. Laura Bachrach, Pamela Steele, and Anne Hiegel for their participation as members of our Data Safety Monitoring Board, and to Dr. Burks for his input in the writing of this article.

\section{References}

1. Sicherer SH. Epidemiology of food allergy. J Allergy Clin Immunol. 2011; 127:594-602. [PubMed: 21236480]

2. Bock SA, Munoz-Furlong A, Sampson HA. Further fatalities caused by anaphylactic reactions to food, 2001-2006. J Allergy Clin Immunol. 2007 Apr; 119(4):1016-8. [PubMed: 17306354] 
3. Boyce JA, Assa'ad A, Burks AW, Jones SM, Sampson HA, Wood RA, et al. Guidelines for the Diagnosis and Management of Food Allergy in the United States: Report of the NIAID-Sponsored Expert Panel. J Allergy Clin Immunol. 2010; 126:S1-58. [PubMed: 21134576]

4. Clark S, Espinola J, Rudders S, Banerjie A, Camargo C. Frequency of US emergency department visits for food-related acute allergic reactions. J Allergy Clin Immunol. 2011; 127:682-683. [PubMed: 21167574]

5. Branum AM, Lukacs SL. Food Allergy Among Children in the United States. Pediatrics. 2009; 124:1549-1555. [PubMed: 19917585]

6. Jones SM, Pons L, Roberts JL, Scurlock A, Perry T, Kulis M, et al. Clinical efficacy and immune regulation with peanut oral immunotherapy. J Allergy Clin Immunol. 2009; 124:292-300. [PubMed: 19577283]

7. Varshney P, Jones SM, Scurlock A, Perry T, Kemper A, Steele P, et al. A randomized controlled study of peanut oral immunotherapy: Clinical desensitization and modulation of the allergic response. J Allergy Clin Immunol. 2011; 127:654-660. [PubMed: 21377034]

8. Blumchen K, Ulbricht H, Staden U, Dobberstein K, Beschorner J, Oliveira LCL, et al. Oral peanut immunotherapy in children with peanut anaphylaxis. J Allergy Clin Immunol. 2010; 126:83-91. [PubMed: 20542324]

9. Bock S, Sampson H, Atkins F, Zeiger R, Lehrer S, Sachs M, et al. Double-blind, placebo-controlled food challenge (DBPCFC) as an office procedure: A manual. J Allergy Clin Immunol. 1988; 82:986-997. [PubMed: 3060514]

10. Hofmann AM, Scurlock AM, Jones SM, Palmer K, Lokhnygina Y, Steele P, et al. Safety of a peanut oral immunotherapy protocol in children with peanut allergy. J Allergy Clin Immunol. 2009; 124:286-291. [PubMed: 19477496]

11. Thyagarajan A, Varshney P, Jones SM, Sicherer S, Wood R, Vickery B, et al. Peanut oral immunotherapy is not ready for clinical use. J Allergy Clin Immunol. 2010; 126:31-32. [PubMed: 20620564]

12. Katz Y, Goldberg M, Stein M, Levy M. Oral immunotherapy: ready for prime time? J Allergy Clin Immunol. 2011; 126:289-290. [PubMed: 21040954]

13. Wasserman RL, Sugerman R, Mireku-Akomeah N, Mansfield L, Baker J. Office-based oral immunotherapy for food allergy is safe and effective. J Allergy Clin Immunol. 2011; 127:290 291. [PubMed: 21040956]

14. Thyagarajan A, Varshney P, Jones SM, Sicherer S, Wood R, Vickery B, et al. Reply. J Allergy Clin Immunol. 2011; 127:291-292. 


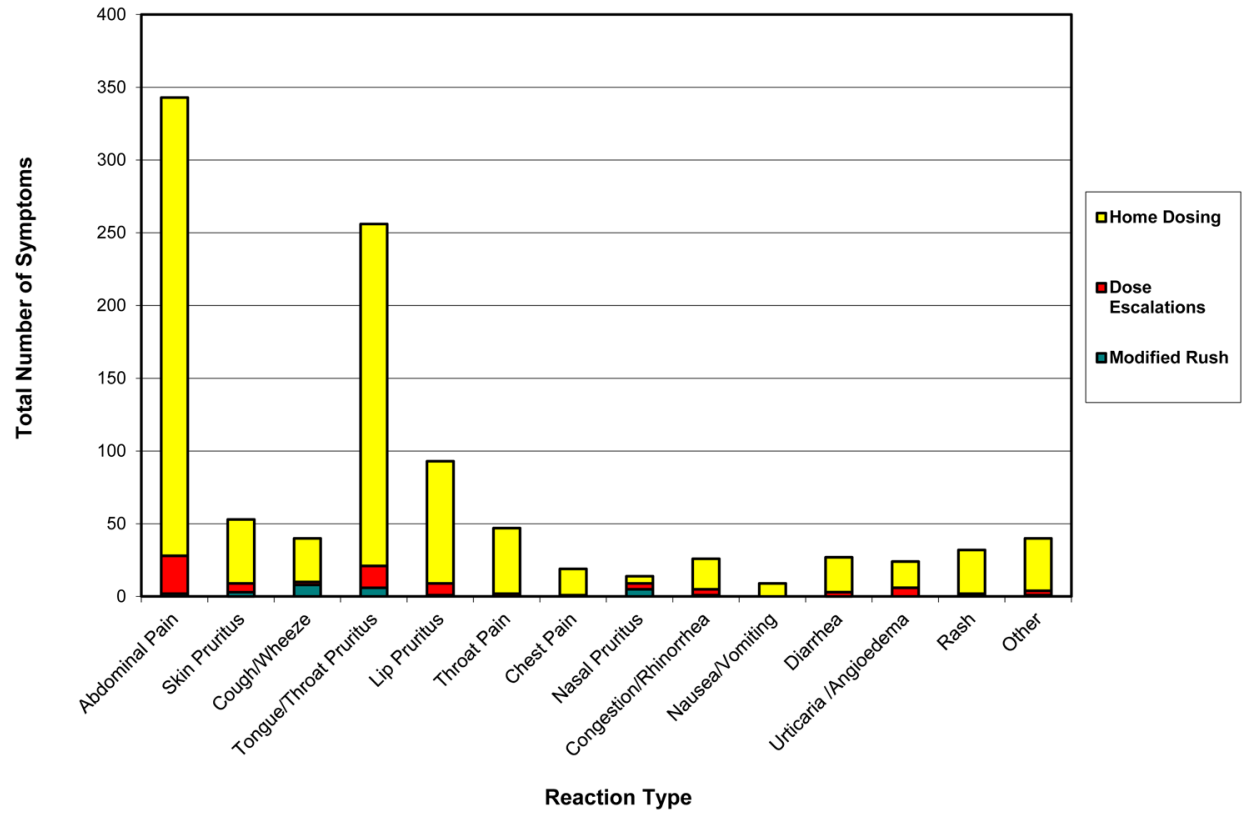

Fig. 1.

Total number of symptoms are depicted by type of symptom and in what situation the reaction occurred (during the modified rush day, during a dose escalation appointment or at home). 


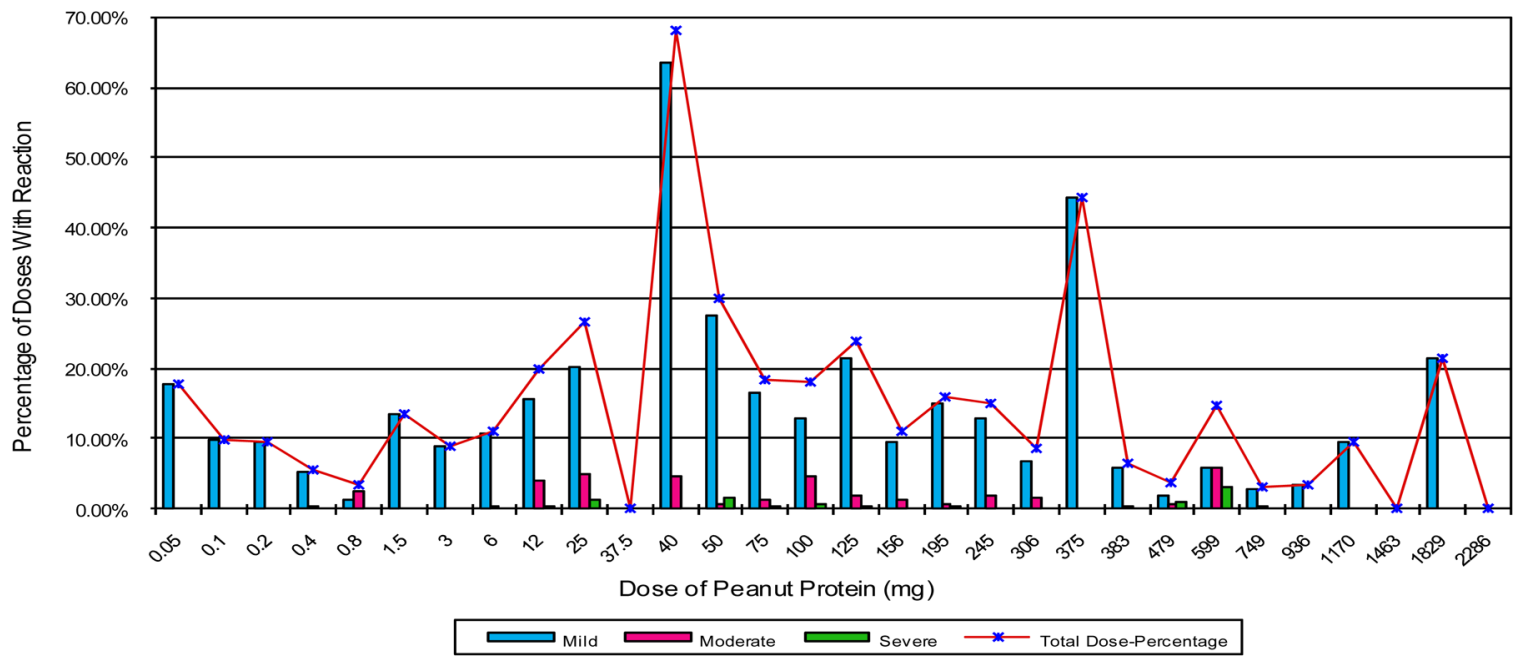

Fig. 2.

The above graph demonstrates the percentage of doses at each dose level that resulted in any reaction (line). Reactions are categorized by the patient (for home doses) or practitioner (for doses in the CTRU) as mild, moderate, or severe. (8) Doses on the $\mathrm{X}$ axis are in milligrams of peanut protein. 


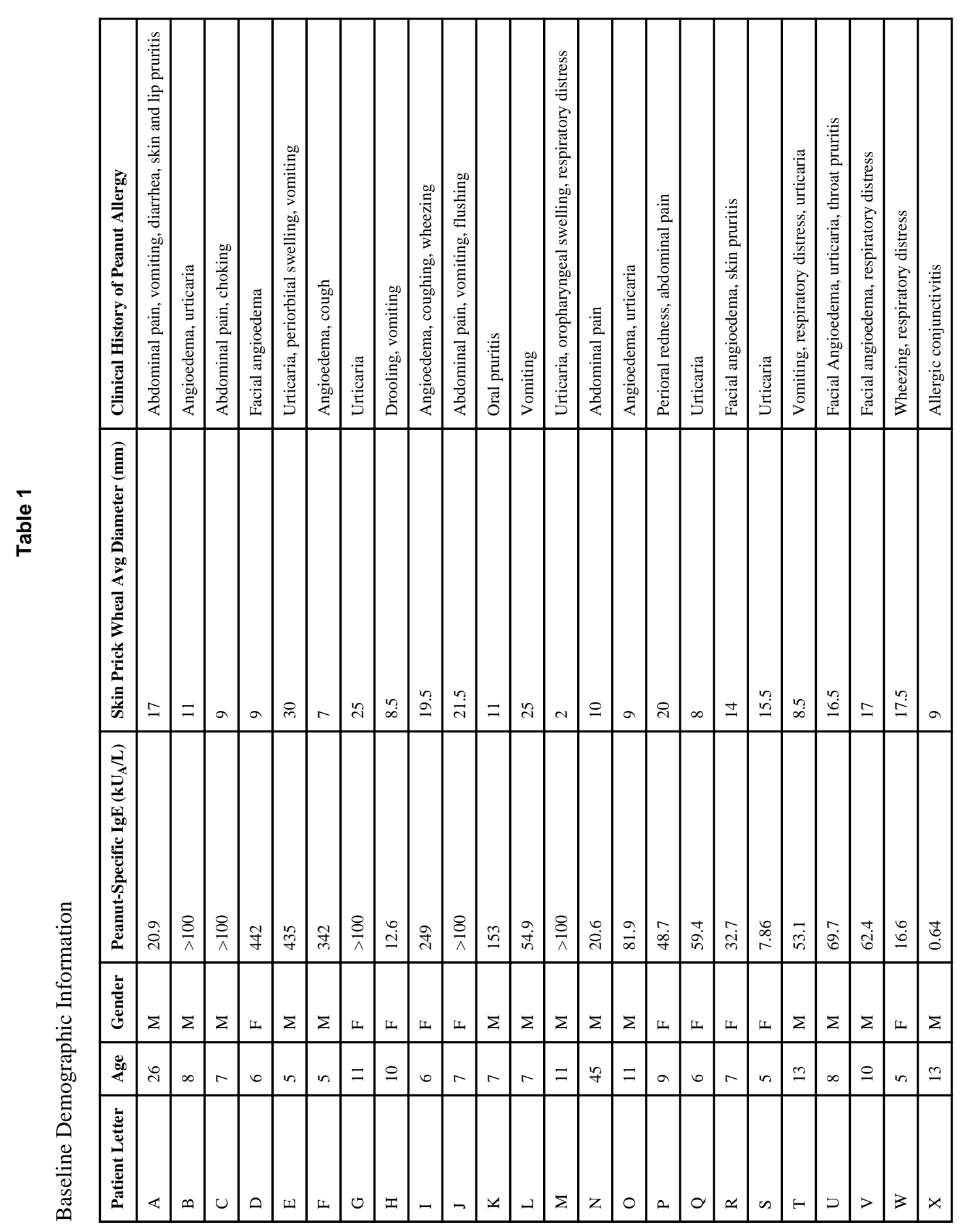

